\title{
Pulmonary embolism secondary to anomalies of deep venous system of the leg
}

\author{
J. FEDERMAN, S. T. ANDERSON, D. S. ROSENGARTEN, \\ AND A. PITT \\ From the Cardiovascular Diagnostic Service, Alfred Hospital, Melbourne
}

Two cases of recurrent pulmonary emboli secondary to thrombosis in anomalies of the deep veins of the lower limb are presented. In both cases the source of emboli was not evident clinically, and it was only after venography that the venous anomalies were discovered, both being confined to one limb and amenable to surgical intervention. Such anomalies have not previously been reported as sources of venous thromboembolism in adults.

The importance of venography in establishing the source of pulmonary emboli, especially when recurrent, is stressed.

The most common source of pulmonary emboli is leg vein thrombosis (Sevitt, 1965). Numerous factors predispose to thromboembolism including immobilisation after injury, surgery, and in the post-partum period (McIntyre et al., 1972). Varicosities of the superficial veins of the lower limbs may also be associated with thromboembolism (Abramson, 1974). Anomalies of the deep venous system of the lower limb are rare, and 2 cases in which these proved to be the source of recurrent pulmonary embolism are presented.

\section{CASE 1}

Five days before admission a 37-year-old woman had suddenly developed retrosternal chest pain while walking. The pain radiated to the left shoulder and back but had no features to suggest a pleuritic or pericardial element and gradually passed off over several hours. Recovery appeared complete apart from some mild lethargy. On the day of admission, after a short period of nausea, the patient noted a fluttering in the chest before collapsing with loss of consciousness for several minutes. There was no significant past or family history and the patient denied taking any drugs.

On admission the patient was pale and clammy with a blood pressure $130 / 100 \mathrm{mmHg}$, pulse 100 per minute, temperature $36.8^{\circ} \mathrm{C}$ and regular respiratory rate of 20 a minute. Cardiovascular examination showed a raised jugular venous pressure of $3 \mathrm{~cm}$, a fourth heart sound, and an ejection systolic murmur along the left sternal edge. Fine inspiratory Received for publication 9 July 1976 crepitations were present at both lung bases and mild bilateral ankle oedema was present. The pulmonary component of the second heart sound did not appear increased and no abnormality was detected in the abdomen. The lower limbs appeared normal with no evidence of venous thrombosis, varicose veins, or vascular bruits. Chest radiograph was normal and electrocardiogram showed a normal frontal axis with non-diagnostic ST-T changes inferolaterally.

As myocardial ischaemia or infarction was considered possible, she was monitored in the coronary care unit. No anticoagulants were given and serial electrocardiograms from the 2nd to 8 th days showed evolving $T$ wave inversion inferiorly and over all the praecordial leads. Apart from an initially raised lactic dehydrogenase of $516 \mathrm{IU} / 1$ (normal < 470) all enzymes were normal. On the 3 rd day a chest radiograph showed the development of an area of opacification in the lateral segment of the right upper lung suggestive of a pulmonary infarct.

Technetium-99-macroaggregated ferrous hydroxide (MAFH) lung scintigram (Fig. 1) showed perfusion defects in both midzones, right apex, and right base, consistent with pulmonary emboli. Shortly after the lung scintigram, the patient had a respiratory arrest with an associated junctional rhythm at a rate of 65 beats a minute. After resuscitation a loud fourth heart sound with an associated right ventricular impulse was noted. The patient was started on full anticoagulation with intravenous heparin and her condition rapidly stabilised. Bilateral lower limb venography was 


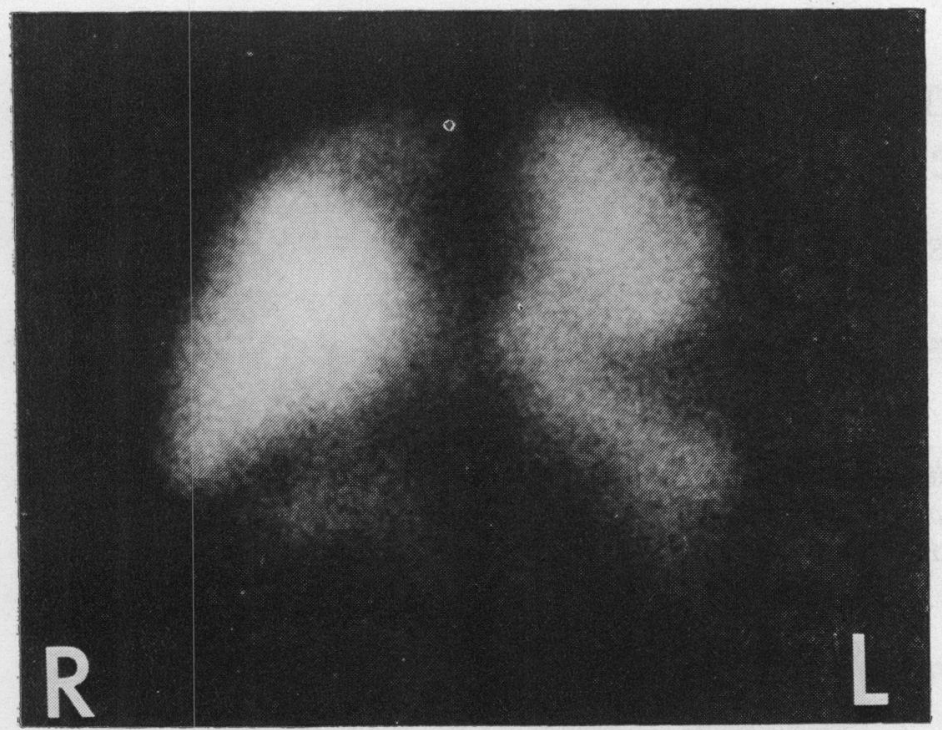

Fig. 1 Lung scintigram in case 1 showing perfusion defects at apex midzone and base of right lung and midzone of left lung.

performed and showed an aneurysmal varix of the right popliteal vein with a filling defect within the varix suggestive of thombus (Fig. 2). Venograph of the left leg was normal.

The next day the popliteal vein was explored under general anaesthesia and an aneurysmal varix was exposed and found to have no arterial connections. The varix was opened, a large thrombus removed, and the varix excised. The defect in the popliteal vein was closed by direct suture. After operation she was maintained on full heparinisation with a calf stimulator being applied to the leg to maintain a high venous flow. Venography was repeated 24 hours later and at the adductor canal showed some compression of the popliteal vein lumen which was thought to be the result of a wound haematoma.

Further venography was performed 10 days after operation because of persistent pain and oedema of the leg. This showed complete obstruction of the deep venous system of the right leg. An inferior venocavogram showed no extension of thrombus beyond the femoral vein. The patient was discharged 3 weeks after operation on oral anticoagulants and full length elastic stockings for persistent leg oedema. Repeat lung scan 3 months later was normal and at 12 months follow-up she remains well with no recurrence of pulmonary emboli.

Fig. 2 Venogram of right leg in case 1 showing an aneurysmal varix of the popliteal vein with intraluminal filling defect (arrow).

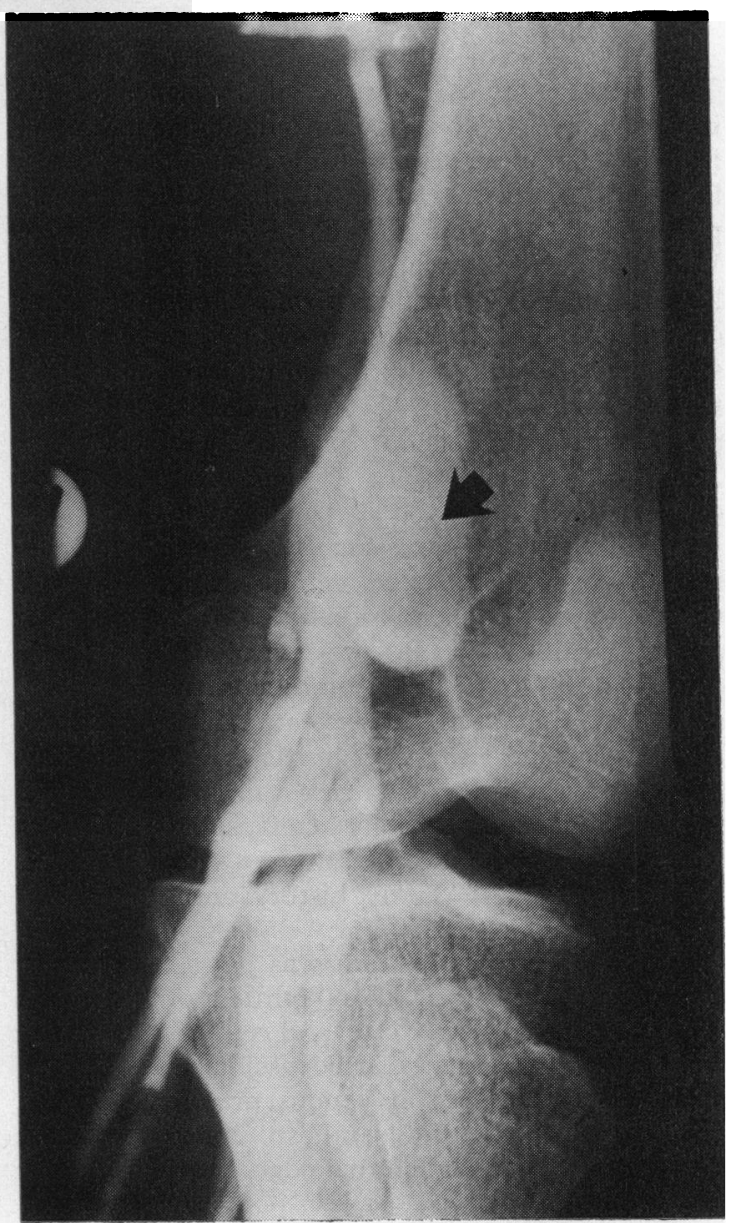




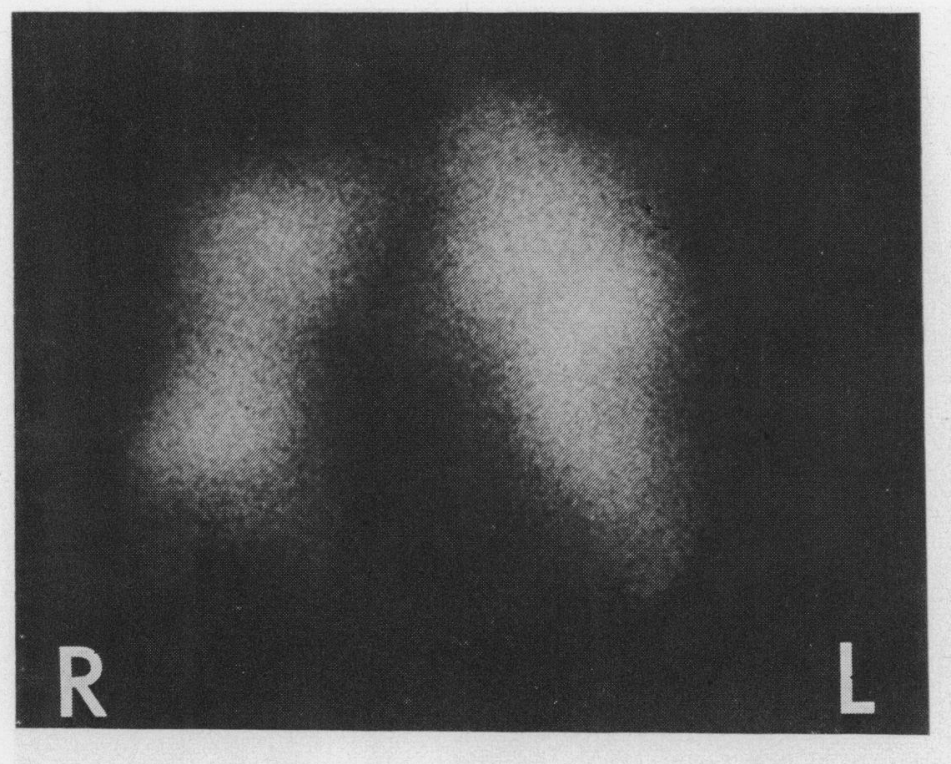

Fig. 3 Lung scintigram in case 2 showing multiple perfusion defects in both lung fields.

CASE 2

A 62-year-old man was referred from another hospital with a 7-month history of progressive effort dyspnoea and recurrent palpitations. Atrial tachycardia had been documented on two occasions. Occasional chest tightness had occurred and for 2 months ankle oedema and abdominal swelling had been noted. His past history included an admission to a country base hospital 10 years previously, with a diagnosis of myocardial infarction said to be complicated by venous thrombosis. The records of this admission were unavailable; however, the patient recalls being dyspnoeic at the time but without chest pain.

Physical examination revealed a patient in no distress with findings of pulmonary hypertension. $\mathrm{He}$ had a raised jugular venous pressure $(2 \mathrm{~cm})$ with a prominent ' $a$ ' wave, a right ventricular lift, and a loud pulmonary component of the second heart sound. There was a pansystolic murmur audible at at lower left sternal edge which increased on inspiration and was consistent with tricuspid regurgitation. Two centimetres of non-pulsatile liver was palpable and his chest was clinically clear. Examination of the legs showed no venous abnormalities or bruits.

A chest $x$-ray film showed cardiomegaly with prominent pulmonary arteries suggesting pulmonary hypertension. An electrocardiogram showed sinus rhythm with right axis deviation of $+110^{\circ}$, right atrial enlargement, clockwise rotation, and digoxin effect. A lung scintigram with Technetium 99 MAFH showed multiple perfusion defects
(Fig. 3). An echocardiogram showed a large right ventricle with a prominent $B$ wave on the tricuspid valve suggesting raised end-diastolic right ventricular pressure with the left ventricle, mitral valve, and aortic valve being normal. Bilateral venography of the lower limbs showed a large varix consisting of numerous small venous channels at the junction of the left femoral and popliteal veins (Fig. 4A). The right leg was normal. The varix contained multiple filling defects thought to be thrombus. Right heart catheterisation (Table) showed pronounced pulmonary hypertension and pulmonary angiography showed multiple filling defects predominantly in the right lung consistent with emboli (Fig. 5).

\section{Table Results of cardiac catheterisation}

\begin{tabular}{lll}
\hline Site & Pressure $(\mathrm{mmHg})$ & Mean pressure $(\mathrm{mmHg})$ \\
\hline Right atrium & a 13 & 6 \\
v 9 & \\
Right ventricle & $90 / 0(15)$ & \\
Pulmonary artery & $90 / 39$ & 53 \\
\hline
\end{tabular}

In view of the extensive nature of the venous anomaly it was decided to ligate the right femoral vein distal to its junction with the long saphenous vein. The patient was maintained on oral anticoagulants and when reviewed 12 months later had shown no improvement. There were signs of persistent pulmonary hypertension and tricuspid regurgitation. Repeat lung scintigram was unchanged. Right heart catheterisation showed a rise in pulmonary artery pressure at $92 / 46 \mathrm{mmHg}$. 


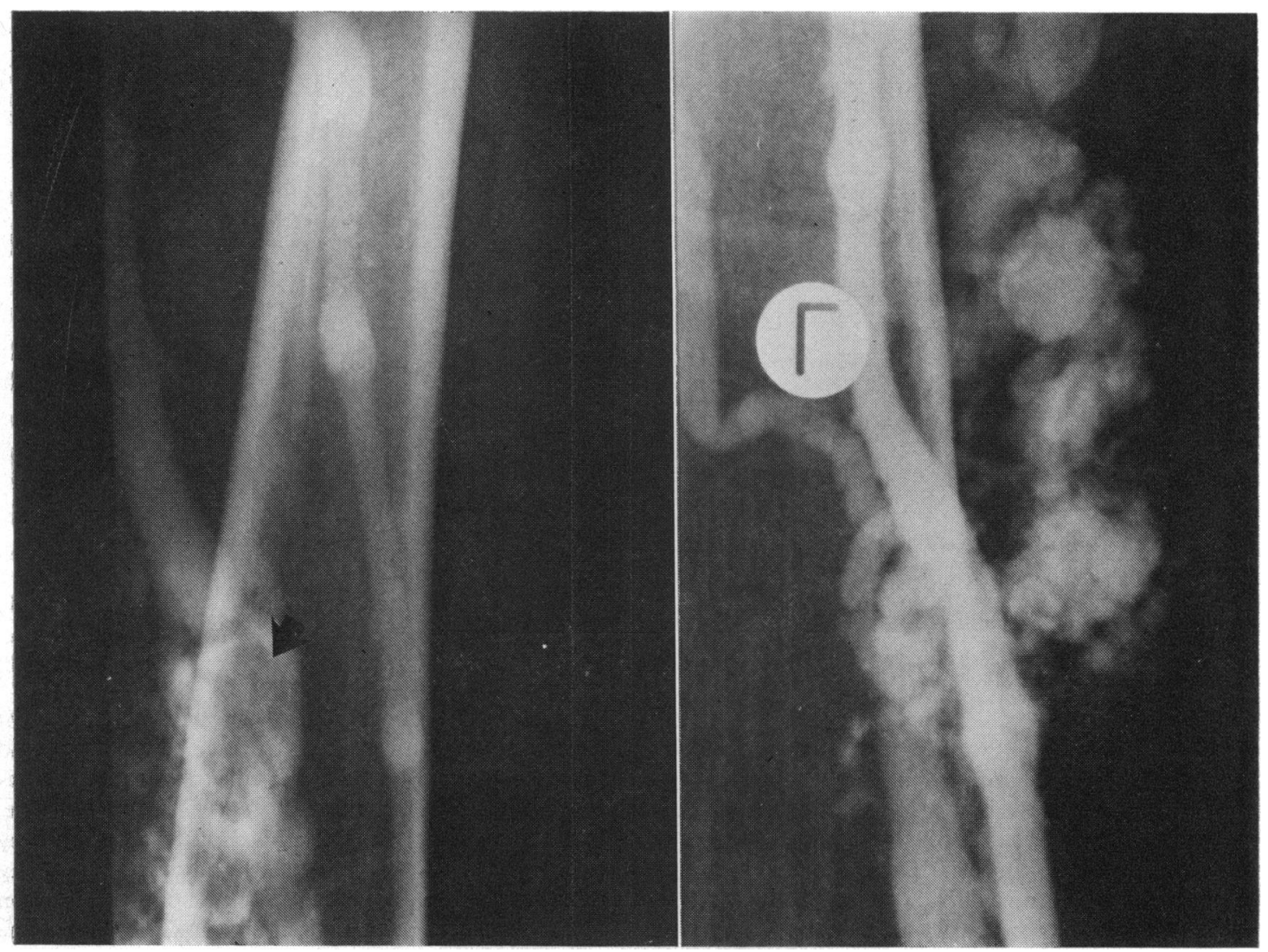

Fig. 4 Venogram of left leg in case 2. (A) The venous angioma with negative shadows suggests intraluminal filling defects (arrow). (B) The repeat venogram 12 months later shows no filling defects within the angioma.

Right atrial pressure had increased, with a mean pressure of $19 \mathrm{mmHg}$. Repeat venography showed opacification of the venous malformation with no filling defects present (Fig. 4B). However, there was interruption to flow in the femoral vein proximally at the site of previous ligation. It was felt that no further surgical procedure was indicated and he was discharged on long-term anticoagulants and antifailure therapy.

\section{Discussion}

Both cases presented with problems associated with pulmonary thromboembolism and were found to have underlying deep venous anomalies which were unsuspected clinically. The first patient presented after an episode of acute thromboembolism which was initially misdiagnosed as an episode of myocardial ischaemia. Chest $x$-ray changes suggested pulmonary infarction and lung scintigram strongly supported pulmonary embolism. Clinical examina- tion gave no clue as to the origin of the emboli and the aneurysmal varix of the right popliteal vein was only discovered as a result of bilateral venography.

The second patient presented a problem of pulmonary hypertension and right heart failure secondary to chronic pulmonary embolism. The history possibly dated back 10 years to when he was thought to have had a myocardial infarct. In this patient pulmonary embolism was confirmed on lung scintigram and pulmonary angiography, with pronounced pulmonary hypertension being present at right heart catheterisation. Again the underlying deep venous varix of the left leg was unsuspected clinically and was only discovered at venography.

Pure venous dysplasias of the extremities are rare and are usually unilateral (Browse, 1973). Malan and Puglionisi (1964) discuss the possible aetiology of pure venous dysplasias and suggest a classification into four main groups: (i) phlebectasia, (ii) phlebangiomas, (iii) phlebangiomatosis; and (iv) a mixed group. Their classification is an extensive one 


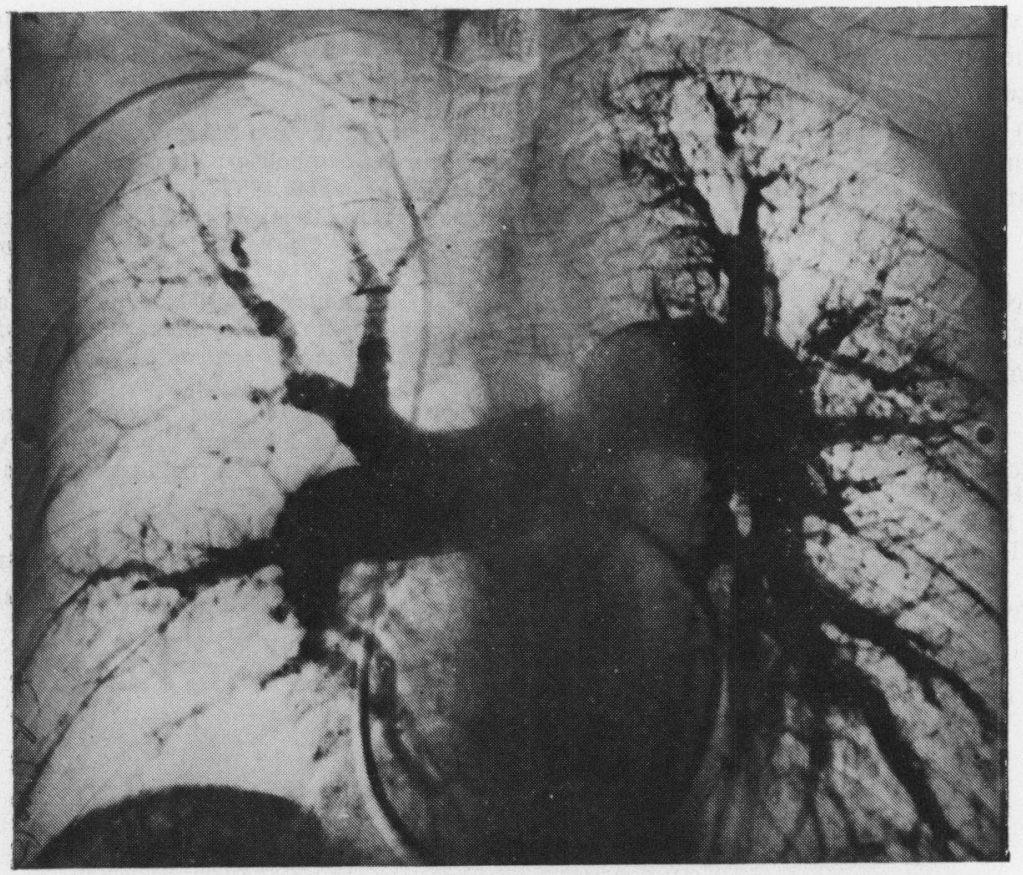

Fig. 5 Pulmonary angiogram in case 2 showing extensive perfusion defects in both lung fields.

but in essence phlebectasia refers to a congenital dilatation of the venous tree of a limb which may be regional or diffuse. Phlebangiomata are limited venous hamartomata with a cavernous and capillary structure. When these venous hamartomata are extensive or multicentred they are called phlebangiomatosis. Associations between the previous three groups constitute the mixed group. They described 21 cases, but in all patients an abnormality of the superficial venous system was present. In 1971 Lea Thomas and Andress described angiographic techniques in venous dysplasias and attempted to simplify the classification on a radiological basis into two main groups. Venous angioma referred to lesions which consist mainly of cavernous spaces while phlebectasia described lesions in which the principal abnormality was dilatation of the venous trunk. They described 16 cases, but again all had superficial venous abnormalities. This makes classification of our cases difficult, since in both there was a localised lesion in the deep venous system with no abnormality of the superficial venous system. Using Lea Thomas and Andress's classification our first patient's lesion most closely resembles a localised phlebectasia and our second patient's lesion a venous angioma.

To our knowledge pulmonary embolism secondary to unsuspected venous anomalies of the deep veins of the lower limb has not previously been described in adults. Maszkiewitz in 1973 did, how- ever, describe a case of a newborn infant who succumbed to pulmonary embolism from thrombi originating from a hypoplastic femoral vein.

Venous thromboembolism constitutes an important cause of morbidity and mortality and several techniques have been developed to aid in the diagnosis. Kakkar in a recent review (1975) stresses the importance of venography as being the single most useful investigation in the diagnosis of suspected thrombosis. It not only helps in outlining the exact site and extent of the thrombus but aids in the decision about subsequent therapy. Other tests such as ${ }^{125}$ I fibrinogen scanning, ultrasound, and plethysmography have been useful as screening tests but would have been of no use in defining accurately the source of embolism in our 2 cases. Venography on the other hand accurately outlined the previously unsuspected venous anomalies and allowed a decision to be made about surgical treatment. In our first case the aneurysmal varix was unilateral and appeared readily suitable for local surgical excision. The defect was closed by direct suture but in view of the narrowing on repeat venography and subsequent secondary thrombosis the use of a vein patch to close the defect or alternatively proximal femoral vein ligation might have prevented this complication. In the second case venography indicated an extensive deep venous varix which was unsuitable for local venous reconstruction and it was decided to ligate the deep femoral vein just distal to the 
origin of the long saphenous vein. This patient's pulmonary hypertension remained unchanged when reviewed 12 months after surgery.

Our 2 cases demonstrate an unusual cause for recurrent pulmonary emboli and emphasise the importance of venography in investigation of this problem.

We thank Dr. J. M. Gardiner for referring our first case and Drs. H. Luke and L. Dugdale for the radiological and isotope imaging studies.

\section{References}

Abramson, D. I. (1974). Vascular Disorders of the Extremities, 2nd ed., p. 551. Harper and Row, Hagerstown, Md.

Browse, N. L. (1973). The veins. In Peripheral Vascular Surgery, p. 127. Ed. by Martin Birnstingl. Heinemann, London.

Kakkar, V. V. (1975). Deep vein thrombosis. Detection and prevention. Circulation, 51, 8-19.
Lea Thomas, M., and Andress, M. R. (1971). Angiography in venous dysplasias of the limbs. American fournal of Roentgenology, Radium Therapy and Nuclear Medicine, 113, 722-731.

McIntyre, K. M., Sasahara, A. A., and Sharma, G. V. R. K. (1972). Pulmonary thromboembolism: current concepts. Advances in Internal Medicine, 18, 199-218.

Malan, E., and Puglionisi, A. (1964). Congenital angiodysplasias of the extremities. Fournal of Cardiovascular Surgery, 5, 87-130.

Maszkiewicz, W. (1973). Pulmonary arterial embolism as a cause of death of a newborn with developmental anomaly of femoral vein. (In Polish.) Polski Tygodnik Lekaraski, 28, 1605-1606.

Sevitt, S. (1965). Anticoagulant prophylaxis against venous thrombosis and pulmonary embolism. In Pulmonary Embolic Disease, p. 265. Ed. by A. A. Sasahara and M. Stein. Grune and Stratton, New York.

Requests for reprints to Dr. A. Pitt, Cardiovascular Diagnostic Service, Alfred Hospital, Commercial Road, Prahran, Victoria 3181, Australia. 